\title{
Field Enhancement in a Grounded Dielectric Slab by Using a Single Superstrate Layer
}

\author{
Constantinos A. Valagiannopoulos ${ }^{1}$ and Nikolaos L. Tsitsas ${ }^{2}$ \\ ${ }^{1}$ Department of Radio Science and Engineering, School of Electrical Engineering, Aalto University, Otakaari 5A Street, \\ 02150 Espoo, Finland \\ ${ }^{2}$ Department of Informatics, Aristotle University of Thessaloniki, 54124 Thessaloniki, Greece
}

Correspondence should be addressed to Nikolaos L. Tsitsas, ntsitsas@csd.auth.gr

Received 19 June 2012; Revised 22 September 2012; Accepted 24 September 2012

Academic Editor: Natalia M. Litchinitser

Copyright ( $) 2012$ C. A. Valagiannopoulos and N. L. Tsitsas. This is an open access article distributed under the Creative Commons Attribution License, which permits unrestricted use, distribution, and reproduction in any medium, provided the original work is properly cited.

\begin{abstract}
The addition of a dielectric layer on a slab configuration is frequently utilized in various electromagnetic devices in order to give them certain desired operational characteristics. In this work, we consider a grounded dielectric film-slab, which is externally excited by a normally-incident Gaussian beam. On top of the film-slab, we use an additional suitably selected single isotropic superstrate layer in order to increase the field concentration inside the slab and hence achieve optimal power transfer from the external source to the internal region. We define a quantity of interest, called "enhancement factor," expressing the increase of the field concentration in the film-slab when the superstrate is present compared to the case that it is absent. It is shown that large enhancement factor values may be achieved by choosing properly the permittivity, the permeability, and the thickness of the superstrate. In particular, it is demonstrated that the field in the film-slab is significantly enhanced when the slab is composed by an $\epsilon$-near-zero (ENZ) or low-index metamaterial.
\end{abstract}

\section{Introduction}

Increased field intensity in a localized area is required in a variety of applications from simple traditional implementations to complex state-of-the-art experiments. Indicatively, a resonance plasmon mode, characterized by a substantial local electric field enhancement, has been reported to be formed between a gold nanorod and an infinite slab in infrared range [1]. In addition, the optical trapping force on a spherical dielectric particle for an arbitrarily positioned focused beam has been demonstrated in [2], by using the generalized vector diffraction theory. Moreover, when considering a simple, analytically solvable cylindrical configuration, it has been shown that optical vortices appear which can be used to stably trap particles of particular sizes and index contrasts with the background [3]. Field enhancement of incident near-infrared light has been also investigated in [4], by using the exhibited surface plasmon polariton from erbium ions in a golden film.
On the other hand, layered, dielectric slab configurations are commonly used in electromagnetic (EM) devices since they possess certain functional advantages such as conformability and ease of fabrication. In particular, dielectric layers, with carefully selected physical and geometrical parameters, are extensively employed to lend particular beneficial characteristics to the considered devices. In [5], a multilayered dielectric coating has been used in semiconductor laser diode optical amplifiers to reduce the reflection coefficient. Furthermore, a discontinuous parallel-plate waveguide acquiring highly selective frequency features through a narrow rectangular layer, filled with axially anisotropic media, has been proposed in [6]. The effect of a metal cladding on the band structure with a two-dimensional photonic crystal slab has been also analyzed in [7]; several types of claddings were used and the way that each cladding changes the background crystal has been identified. The fact that a single isotropic (idealized) cloaking layer may successfully suppress the dominant scattering coefficients of moderately 
thin elongated objects, even for finite lengths comparable with the incident wavelength, has been pointed out in [8]. Finally, in [9], an additional cloaking dielectric layer has been exploited in a microstrip receiving antenna in order to render it as low profile as possible and also mitigate its EM response.

In this work, we combine the two aforementioned concepts, namely, the potential field enhancement by the addition of extra layers, in order to examine possible improvements offered in a relatively simple scattering configuration. More precisely, we consider the basic structure of a perfect electric conducting (PEC) grounded dielectric filmslab, which is frequently employed in several EM devices, see for example, $[10,11]$. The slab structure is externally excited by a normally incident Gaussian beam; such an incident wave is commonly considered in similar configurations [12]. On the top of the dielectric film-slab, we use an additional superstrate layer, with controllable permittivity and permeability, in order to increase the induced field concentration inside the original film-slab. In this way, we seek to achieve an optimal transfer of the EM power from the external source to the internal region, where enhanced intensity is required. We solve semi-analytically the related boundary-value problem, and then define a basic quantity of interest expressing how large is the field concentration in the film-slab when the superstrate layer is present compared to the case when it is absent; this quantity is called "enhancement factor." The variations of the enhancement factor with respect to the operating frequency and to the physical and geometrical characteristics of the structure are depicted. It is shown that it is possible to achieve large enhancement factor values by choosing properly the permittivity, the permeability, and the thickness of the superstrate layer. Particularly, it is demonstrated that the film-slab's field is significantly enhanced when the slab is composed by an $\epsilon$-near-zero (ENZ) or low-index metamaterial.

\section{Geometrical Configuration and Incident Field}

The under consideration two-dimensional (2D) configuration, as well as the respective Cartesian $(x, y, z)$ coordinate system, are depicted in Figure 1. The configuration is comprised of a perfect electric conductor (PEC) plane at $y=L+w_{1}$ covered by a dielectric film-slab occupying the area $L<y<L+w_{1}$ (region 1). The film-slab is filled by a magnetically inert material with complex relative dielectric permittivity $\epsilon_{r 1}$. The structure of the PEC plane and the film-slab is additionally covered by a slab superstrate (region 2) of thickness $w_{2}<L$ and relative intrinsic physical parameters $\epsilon_{r 2}$ and $\mu_{r 2}$. The infinite plane region $y<L-w_{2}$ (vacuum region 0 ) above the superstrate is characterized by permittivity $\epsilon_{0}$ and permeability $\mu_{0}$. The entire structure is assumed uniform along the direction $\hat{\mathbf{z}}$.

The adopted time dependence is of the form $\exp (j \omega t)$, with $\omega=2 \pi f$ being the angular frequency, and is being suppressed throughout hereinafter. Under this consideration, the imaginary part of the permittivity $\epsilon_{r 1}$ is negative

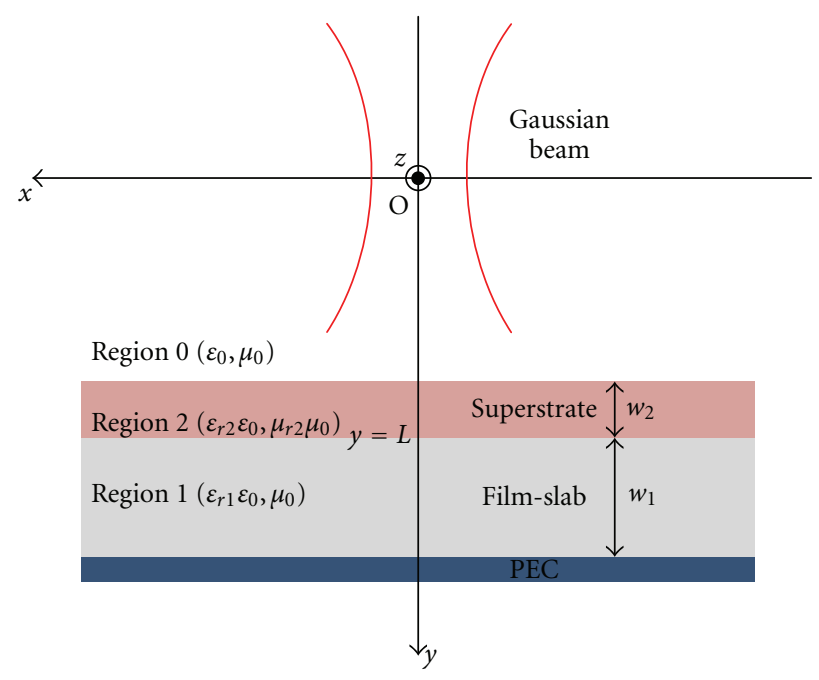

FIgURE 1: Geometrical configuration of the 2D structure under investigation composed of a planar dielectric film-slab, lying between a PEC plane and a superstrate. The structure is excited externally by a Gaussian beam.

(corresponding to passive materials). The remaining relative material parameters $\epsilon_{r 2}$ and $\mu_{r 2}$ are real.

A Gaussian beam, with its source at the origin $O$ of the Cartesian coordinate system, illuminates normally the above described planar configuration. This incident Gaussian electric field is $z$-polarized and propagates along the $y$-axis (as will be analyzed below in details). Hence, due to the 2D nature of the configuration, all reflected and refracted electric fields in regions 0,1 , and 2 will also be $z$-polarized.

The aim of this work is to select suitable geometrical and physical parameters of the superstrate layer $\left(w_{2}, \epsilon_{r 2}, \mu_{r 2}\right)$ in order to enhance the intensity of the field induced in the filmslab region 1 by the external Gaussian beam excitation. More precisely, we require the EM field concentration in region 1 to become as large as possible in order to make it feasible for this "trapped" EM energy to be processed efficiently and be subsequently utilized for relevant applications, like for example, the ones pointed out above in the Introduction; see [1-9]. It is worth to note that we are interested only in the potential enhancement of the induced EM energy in the film-slab and not in the investigation of surface guided waves that may propagate in the infinite slab. For this reason, we consider that the slab is composed of a lossy material, characterized by a complex permittivity $\epsilon_{r 1}$, hence constituting waves in the film-slab to become evanescent.

\section{Analysis of the Incident Gaussian Beam}

3.1. Approximate Expression of the Beam's Envelope. The electric field of an incident Gaussian beam propagating in vacuum along the $+y$-axis is expressed as follows:

$$
\mathbf{E}_{0, \text { inc }}(x, y)=\hat{\mathbf{z}} \Pi(x, y) \exp \left(-j k_{0} y\right), \quad y>0,
$$

where $k_{0}=\omega \sqrt{\epsilon_{0} \mu_{0}}$ is the free space wavenumber, while $\Pi(x, y)$ is the envelope of the beam. It is reasonable to 
consider that $\Pi(x, y)$ is described by an exponential function involving the product of a complex function $\beta(y)$ with a $-x^{2}$ factor; the latter assures the Gaussian nature of the beam. Hence, we conclude to the expression

$$
\Pi(x, y)=A(y) \exp \left[-\beta(y) x^{2}\right]
$$

where $A(y)$ is a complex amplitude function, while $\mathfrak{R}[\beta(y)] \geq 0$.

Next, in order to determine, by analytical means, approximate expressions for $A(y)$ and $\beta(y)$, we substitute expression (1) of $\mathbf{E}_{0 \text {,inc }}(x, y)$ into the vector Helmholtz equation for vacuum region 0 and assume that the term proportional to $\partial^{2} \Pi / \partial y^{2}$ is negligible, since the main contribution with respect to $y$ in the electric field is contained in the term $\exp \left(-j k_{0} y\right)$. In this way, we result to two first-order coupled nonlinear ordinary differential equations, which are solved exactly yielding $[13,14]$

$$
\begin{gathered}
\beta^{\prime}(y)+\frac{2}{j k_{0}} \beta^{2}(y)=0 \Longrightarrow \beta(y)=-\frac{k_{0}}{k_{0} S+2 j y}, \\
A^{\prime}(y)+\frac{\beta(y)}{j k_{0}} A(y)=0 \Longrightarrow A(y)=\frac{1}{\sqrt{k_{0} S+2 j y}},
\end{gathered}
$$

where $S$ is an arbitrary coefficient measured in area units. We consider that $S=-2 \chi / k_{0}$, where the parameter $\chi>0$ has length units. By combining (2)-(4) and separating the real and imaginary parts, we find that the envelope function is given by

$$
\begin{aligned}
\Pi(x, y)= & \sqrt[4]{\frac{4 \chi}{R(\chi, y)}} \exp \left[\frac{j}{2} \arctan \left(\frac{y}{\chi}\right)\right] \\
& \times \exp \left[-x^{2} \frac{k_{0}}{R(\chi, y)}\right] \exp \left[-j x^{2} \frac{k_{0}}{R(y, \chi)}\right],
\end{aligned}
$$

where

$$
R(y, \chi)=2 y\left[1+\left(\frac{x}{y}\right)^{2}\right] .
$$

The parameter $\chi$ characterizes the Gaussian beam and will be hereinafter called "concentration length," since it is inversely related to the local power concentration at the source (origin $O$ ). In other words, a large concentration length indicates a slowly decaying behavior of the envelope, as $|x|$ and $|y|$ increase. Representative visualizations of the absolute value of the Gaussian beam's electric field for $\chi=0.1,0.3$, and 0.9 are depicted in Figures 2(a)-2(c), respectively.

3.2. Fourier Integral Expression of the Beam. Let $\mathbf{v}(x, y)=$ $\widehat{\mathbf{z}} v(x, y)$ be a vector function satisfying the Helmholtz equation. We consider the 1D Fourier transform pair of

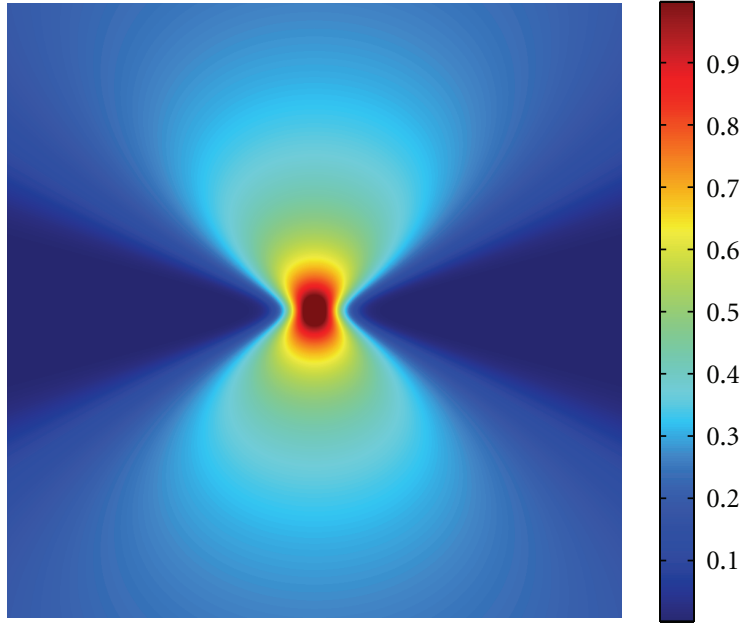

(a)

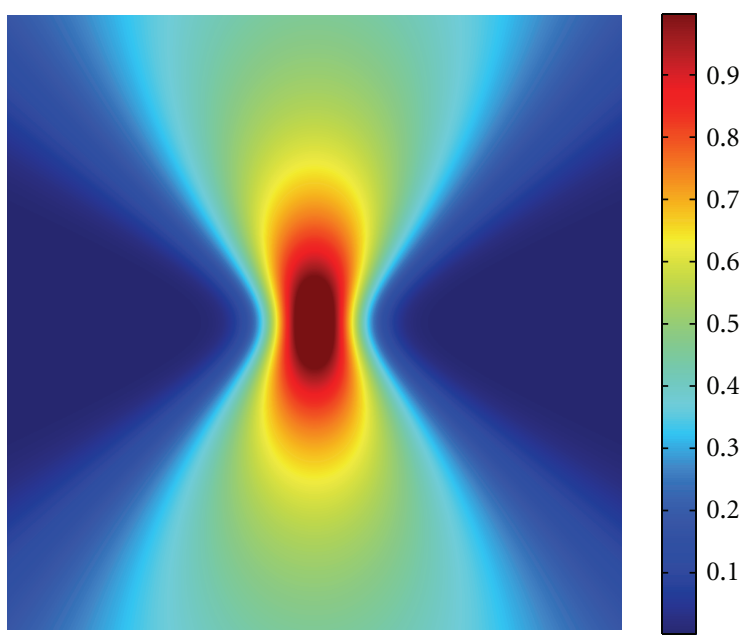

(b)

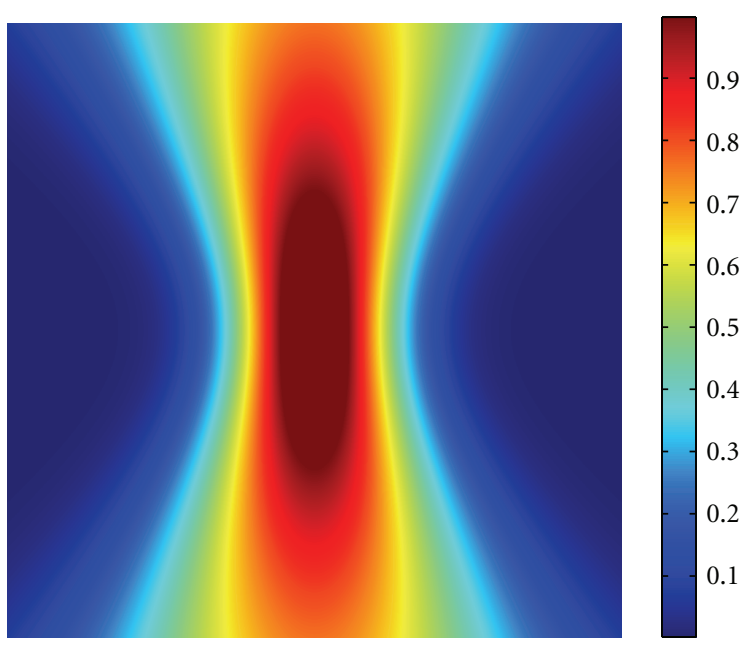

(c)

Figure 2: Contour plots of the absolute value of the Gaussian beam's field given by (1) and (5), for $k_{0}=2 \pi$ and $\chi=0.1,0.3$, and 0.9 , respectively; both axes correspond to arbitrary length units. 
$v(x, 0)$ (namely of the restriction of $v(x, y)$ on $y=0$ ) as folllows:

$$
\begin{aligned}
& v(x, 0)=\frac{1}{2 \pi} \int_{-\infty}^{+\infty} V(\alpha) \exp (j \alpha x) d \alpha, \\
& V(\alpha)=\int_{-\infty}^{+\infty} v(x, 0) \exp (-j \alpha x) d x .
\end{aligned}
$$

Equation (7) expresses a complex function $v(x, 0)$ as a weighted integral of the exponential factors $\exp (j \alpha x)$.

Moreover, if a function $\mathbf{w}(x, y)=\hat{\mathbf{z}} \exp (j \alpha x) w(y)$ satisfies the Helmholtz equation, then it is readily obtained that $w(y)=\exp \left( \pm y \sqrt{\alpha^{2}-k_{0}^{2}}\right)$. Therefore, by taking into account [13]

$$
\int_{-\infty}^{+\infty} \Pi(x, 0) \exp (-j \alpha x) d x=\sqrt[4]{2} \sqrt{\frac{2 \pi \chi}{k_{0}}} \exp \left(-\frac{\chi \alpha^{2}}{2 k_{0}}\right),
$$

where the envelope $\Pi$ is given by (5), and considering that the incident field $\mathbf{E}_{0 \text {,inc }}$, expressed by (1) and (5), approximately satisfies the vector Helmholtz equation, we result to (see also the discussions in [14])

$$
\begin{aligned}
\mathbf{E}_{0, \text { inc }}(x, y)= & \hat{\mathbf{z}} \sqrt[4]{2} \sqrt{\frac{\chi}{2 \pi k_{0}}} \int_{-\infty}^{+\infty} \exp \left(-\frac{\chi \alpha^{2}}{2 k_{0}}\right) \\
& \times \exp \left(j \alpha x-y \sqrt{\alpha^{2}-k_{0}^{2}}\right) d \alpha, \quad y>0 .
\end{aligned}
$$

The square root is evaluated with a positive imaginary part (in case $|\alpha|<k_{0}$ ). We emphasize that (10) is not equivalent to the expression (1) of $\mathbf{E}_{0 \text {,inc }}$ (with $\Pi$ given by (5)), since the latter expression satisfies only approximately the Helmholtz equation (the term $\partial^{2} \Pi / \partial y^{2}$ was assumed negligible).

Now, we assume that an arbitrary 2D layered configuration is excited at $y>0$ with a plane wave of the form:

$$
\mathbf{e}_{\text {inc }}(x, y, \alpha)=\hat{\mathbf{z}} \exp \left(j \alpha x-y \sqrt{\alpha^{2}-k_{0}^{2}}\right),
$$

(which is propagating for $|\alpha|<k_{0}$ and evanescent for $\left.|\alpha|>k_{0}\right)$, and we determine the generated scattered field $\mathbf{e}_{\text {scat }}(x, y, \alpha)$ in each of the layers. Then, the corresponding scattered field due to a Gaussian incident beam is computed by acting with the following operator:

$$
\mathcal{g}\{\mathbf{f}\} \equiv \sqrt[4]{2} \sqrt{\frac{\chi}{2 \pi k_{0}}} \int_{-\infty}^{+\infty} \exp \left(-\frac{\chi \alpha^{2}}{2 k_{0}}\right) \mathbf{f} d \alpha,
$$

on the vector $\mathbf{f}=\mathbf{e}_{\text {scat }}(x, y, \alpha)$. This conclusion is due to the fundamental principle of superposition and the linearity of the Maxwell's equations. The factor $\exp \left(-\chi \alpha^{2} / 2 k_{0}\right)$, appearing in (12), guarantees the convergence of the integral for $|\alpha| \rightarrow+\infty$ (recall that $\chi>0)$.

\section{Solution of the Scattering Problem}

4.1. Auxiliary Problem: Plane Wave Excitation. First, we analyze the auxiliary scattering problem, corresponding to plane wave excitation. To this end, we consider that the layered configuration of Figure 1 is excited by the plane wave

$$
\mathbf{e}_{0, \text { inc }}(x, y ; \alpha)=\hat{\mathbf{z}} \exp \left[j \alpha x-\gamma_{0}(\alpha) y\right],
$$

where the real parameter $\alpha$ determines the direction of propagation; $\alpha=0$ corresponds to normal incidence on the layered structure. The radiation functions $\gamma$ are defined by

$$
\gamma_{u}(\alpha)=\sqrt{\alpha^{2}-k_{0}^{2} \epsilon_{r u} \mu_{r u}}, \quad u=0,1,2
$$

with $\epsilon_{r 0}=\mu_{r 0}=1$.

The electric field in the film-slab region 1 is given by

$$
\mathbf{e}_{1}(x, y ; \alpha)=\widehat{\mathbf{z}} C_{1}(\alpha) \sinh \left[\gamma_{1}(\alpha)\left(y-L-w_{1}\right)\right] \exp (j \alpha x),
$$

where $C_{1}(\alpha)$ is a complex function of $\alpha$. By imposing the related boundary conditions, namely, continuity of the tangential electric and magnetic field components on $y=L$, and the PEC boundary condition on $y=L+w_{1}$, we obtain the following explicit form:

$$
\begin{aligned}
& C_{1}(\alpha)=4 e^{\gamma_{0}(\alpha)\left(w_{2}-L\right)} \mu_{r 2} \gamma_{0}(\alpha) \gamma_{2}(\alpha) \\
& \quad \times\left\{\begin{array}{c}
e^{-\gamma_{2}(\alpha) w_{2}}\left(\mu_{r 2} \gamma_{0}(\alpha)-\gamma_{2}(\alpha)\right) \\
\times\left[\mu_{r 2} \cosh \left(\gamma_{1}(\alpha) w_{1}\right) \gamma_{1}(\alpha)\right. \\
\left.-\sinh \left(\gamma_{1}(\alpha) w_{1}\right) \gamma_{2}(\alpha)\right] \\
-e^{\gamma_{2}(\alpha) w_{2}}\left(\mu_{r 2} \gamma_{0}(\alpha)+\gamma_{2}(\alpha)\right) \\
\times\left[\mu_{r 2} \cosh \left(\gamma_{1}(\alpha) w_{1}\right) \gamma_{1}(\alpha)\right. \\
\left.+\sinh \left(\gamma_{1}(\alpha) w_{1}\right) \gamma_{2}(\alpha)\right]
\end{array}\right\} .
\end{aligned}
$$

Equations (15) and (16) constitute the solution of the scattering problem due to the plane incident wave (13).

Moreover, we are also interested in the case where the superstrate is absent, namely, $\epsilon_{r 2}=\mu_{r 2}=1$ and hence $\gamma_{2}(\alpha)=\gamma_{0}(\alpha)$. For this case the electric field in the film-slab region 1 is given by

$$
\widetilde{\mathbf{e}}_{1}(x, y ; \alpha)=\widehat{\mathbf{z}} \widetilde{C}_{1}(\alpha) \sinh \left[\gamma_{1}(\alpha)\left(y-L-w_{1}\right)\right] \exp (j \alpha x),
$$

where function $\widetilde{C}_{1}(\alpha)$ takes now the following simplified form:

$$
\widetilde{C}_{1}(\alpha)=-\frac{2 e^{-\gamma_{0}(\alpha) L} \gamma_{0}(\alpha)}{\cosh \left(\gamma_{1}(\alpha) w_{1}\right) \gamma_{1}(\alpha)+\sinh \left(\gamma_{1}(\alpha) w_{1}\right) \gamma_{0}(\alpha)}
$$

Equations (17) and (18) provide the solution of the scattering problem due to the plane incident wave (13) impinging on the corresponding configuration of Figure 1 where the superstrate layer is absent.

4.2. Enhancement Factor. Once the plane wave incidence electric field has been determined by means of (15) or (17), the total field induced in region 1, due to the Gaussian beam 
incidence, is computed by using of the operator defined in (12) as follows:

$$
\begin{aligned}
\mathbf{E}_{1}(x, y) & =\mathcal{g}\left\{\mathbf{e}_{1}(x, y ; \alpha)\right\} \\
& =\sqrt[4]{2} \sqrt{\frac{\chi}{2 \pi k_{0}}} \int_{-\infty}^{+\infty} \exp \left(-\frac{\chi \alpha^{2}}{2 k_{0}}\right) \mathbf{e}_{1}(x, y ; \alpha) d \alpha, \\
\widetilde{\mathbf{E}}_{1}(x, y) & =g\left\{\widetilde{\mathbf{e}}_{1}(x, y ; \alpha)\right\} \\
& =\sqrt[4]{2} \sqrt{\frac{\chi}{2 \pi k_{0}}} \int_{-\infty}^{+\infty} \exp \left(-\frac{\chi \alpha^{2}}{2 k_{0}}\right) \tilde{\mathbf{e}}_{1}(x, y ; \alpha) d \alpha .
\end{aligned}
$$

The first and second of the above equations correspond, respectively, to the case with and without the superstrate. Moreover, we remind that the parameter $\chi$ (concentration length) is the only degree of freedom characterizing and describing the incident Gaussian beam.

The integrations in (19) and (20) are carried out by using conventional numerical integration techniques. In particular, in order to exploit the MATLAB matrix-oriented architecture, we use the embedded $\operatorname{trapz}(x, Y)$ function for ordinary trapezoidal integrations which can accept multidimensional arguments. To be more specific, in compiled languages (like $\mathrm{C}++$ ), the loop bodies are transformed into machine code only once at compile time. On the other hand, in interpreted languages (like MATLAB), the loop body is interpreted each time a loop is executed. Therefore, it is preferable to perform vectorized operations in MATLAB for repeated integrations. This is feasible only with $\operatorname{trapz}(x, Y)$, where $Y$ can be a multidimensional matrix, contrary to adaptive MATLAB routines such as quad $(f, a, b)$ where a function $f$ is integrated from $a$ to $b$.

Moreover, we point out that the integrands in (19) and (20) have singularities corresponding to the eigenmode solutions of the slab waveguides formed by the grounded film-slab and the superstrate layer in isolation. However, these singularities are complex since the film-slab has been assumed to be composed of a lossy dielectric material (in order not to deal with possible surface guided waves; see Section 2 above). Hence, they do not contribute to the evaluation of the associated integrals where the integrations are performed on the real axis of the $\alpha$-plane.

Now, in order to estimate the effect of the superstrate layer (region 2) on the total EM power concentrated in region 1 , we introduce the follwing "enhancement factor":

$$
\mathcal{E} \mathcal{F}=\frac{\int_{L}^{L+w_{1}} \int_{-\infty}^{+\infty}\left|\hat{\mathbf{z}} \cdot \mathbf{E}_{1}(x, y)\right|^{2} d x d y}{\int_{L}^{L+w_{1}} \int_{-\infty}^{+\infty}\left|\hat{\mathbf{z}} \cdot \widetilde{\mathbf{E}}_{1}(x, y)\right|^{2} d x d y} .
$$

This factor represents the ratio of the total field's power induced in the film-slab when the superstrate is present over the corresponding power when the superstrate is absent. The beneficial influence of the superstrate layer, leading to an enhanced power concentration, is demonstrated when $\mathcal{E F}>1$.

\section{Numerical Results}

In this Section, we depict and discuss the variations of the enhancement factor $\mathcal{E} \mathcal{F}$ versus the superstrate's characteristics $w_{2}, \epsilon_{r 2}$, and $\mu_{r 2}$ as well as versus the operating frequency $f$. In the following numerical simulations, we choose the material parameters composing the grounded layered slab configuration under consideration according to typical values encountered in similar configurations; see for example, indicatively $[3,4,15]$. In particular, the following parameters remain constant hereinafter (unless stated otherwise): operating frequency $f_{0}=300 \mathrm{THz}$ with corresponding fixed wavelength $\lambda_{0}$, film-slab's thickness $w_{1}=\lambda_{0}$, distance between the source and the film-slab $L=0.5 \lambda_{0}$, and beam's concentration length $\chi=0.1 \lambda_{0}$.

Figures 3(a) and 3(b) show the enhancement factor $\mathcal{E F}$ contour plots with respect to the superstrate's relative dielectric permittivity $\epsilon_{r 2}$ and relative magnetic permeability $\mu_{r 2}$ for $\epsilon_{r 1}=50-5 j$ with (a) $w_{2}=0.1 \lambda_{0}$ and (b) $w_{2}=0.4 \lambda_{0}$. The purpose of examining such contour graphs is to find "operation points" with respect to $\epsilon_{r 2}$ and $\mu_{r 2}$ for which the electric field induced in the film-slab region 1 is considerably enhanced. From Figures 3(a) and 3(b), we observe that such points indeed exist equalling, for example, approximately $\left(\epsilon_{r 2}, \mu_{r 2}\right)=(18,3)$ for $w_{2}=0.1 \lambda_{0}$ and $\left(\epsilon_{r 2}, \mu_{r 2}\right)=(8,1.2)$ for $w_{2}=0.4 \lambda_{0}$. At these two operating points, the $\mathcal{E} \mathcal{F}$ values are increased to nearly $250 \%$, meaning that the power induced in region 1 of the layered configuration of Figure 1 is nearly 2.5 times the power induced in the corresponding superstratefree configuration. We conclude that several choices exist for operating points with large values of $\mathcal{E} \mathcal{F}$; in particular, by comparing Figures $3(\mathrm{a})$ and $3(\mathrm{~b})$, we see that the number of potential operating points increases with the thickness $w_{2}$ of the superstrate. The fact that $\mathcal{E F}$ may take large values for several choices of $\epsilon_{r 2}, \mu_{r 2}$, and $w_{2}$ offers flexibility in designing the structure and choosing appropriate and realizable material values with respect to the applications under consideration.

Moreover, Figures 4(a) and 4(b) depict $\mathscr{E} \mathcal{F}$ as function of the superstrate's relative thickness $w_{2} / L$ for $\epsilon_{r 1}=50-5 j$, $\epsilon_{r 2}=9, \mu_{r 2}=1$ with (a) $L=0.5 \lambda_{0}$, and $\chi / \lambda_{0}=0.01,0.05,0.5$, and (b) $\chi / \lambda_{0}=0.1$, and $L / \lambda_{0}=0.1,0.2,0.3,1$. Figure 4(a) shows that $\mathscr{E} \mathcal{F}$ attains certain maxima with respect to $w_{2}$ and the values of these maxima increase with $w_{2}$. Also, $\mathscr{E F}$ generally decreases with increasing $\chi$, namely, as the incident Gaussian beam becomes less concentrated in the vicinity of the origin $O$. Even for initially very concentrated beams $\left(\right.$ small $\chi$ ) there exist certain thicknesses $w_{2}$ for which $\mathcal{E F}$ takes large values. On the other hand, from Figure 4(b) we see that large values of $\mathscr{E} \mathcal{F}$ may be achieved even for small distances $L$ between the source and the film-slab. This fact could be exploited appropriately, particularly for lasing applications.

Figures 5(a) and 5(b) represent $\mathcal{E F}$ as function of the normalized operating frequency $f / f_{0}$ for $f_{0}=300 \mathrm{THz}$, $\epsilon_{r 1}=50-5 j, w_{2}=0.25 \lambda_{0}$ with (a) $\mu_{r 2}=1$, and $\epsilon_{r 2}=$ $2,5,10,15$, and (b) $\epsilon_{r 2}=5$, and $\mu_{r 2}=1,2,3,4$. In the entire examined frequency band, the values of $\mathcal{E F}$ are larger than 1. Moreover, $\mathcal{E} \mathcal{F}$ is oscillatory with $f$ with the period of the 


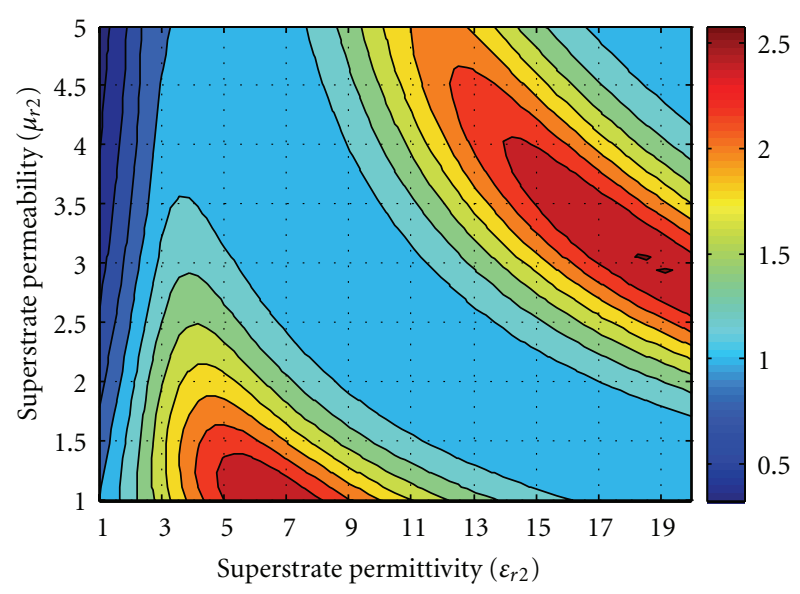

(a)

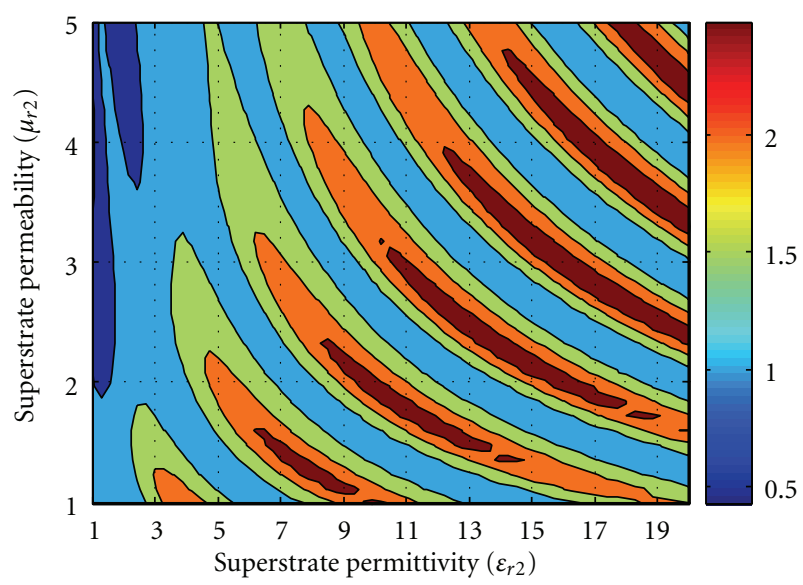

(b)

FIgURE 3: Enhancement factor contour plots as functions of the superstrate's relative dielectric permittivity $\epsilon_{r 2}$ and relative magnetic permeability $\mu_{r 2}$ for $f_{0}=300 \mathrm{THz}, w_{1}=\lambda_{0}, L=0.5 \lambda_{0}, \epsilon_{r 1}=50-5 j$, $\chi=0.1 \lambda_{0}$ with (a) $w_{2}=0.1 \lambda_{0}$ and (b) $w_{2}=0.4 \lambda_{0}$.

oscillations decreasing for increasing $\epsilon_{r 2}\left(\mu_{r 2}\right)$ with constant $\mu_{r 2}\left(\epsilon_{r 2}\right)$.

Also, we point out that additional numerical simulations (not reported here) have demonstrated that the values of $\epsilon_{r 2}$ and $\mu_{r 2}$ giving local maxima of $\mathcal{E} \mathcal{F}$ are independent of the film-slab's permittivity $\epsilon_{r 1}$. Besides, the related maximum values of $\mathcal{E} \mathcal{F}$ increase with $\epsilon_{r 1}$.

Now, we turn our attention to the investigation of the associated EM field concentration phenomena encountered in representative optical applications. To this end, we select realizable material parameter values in the optical region, according to those reported in [16-18]. Figures 6(a) and 6(b) depict the $\mathcal{E} \mathcal{F}$ versus the superstrate's relative (a) permittivity $\epsilon_{r 2}$ for $\mu_{r 2}=1$, and (b) permeability $\mu_{r 2}$ for $\epsilon_{r 2}=2$, with $w_{2}=0.25 \lambda_{0}, \mathfrak{R}\left[\epsilon_{r 1}\right]=-5,-2,-0.1,2,5$, and $\mathfrak{I}\left[\epsilon_{r 1}\right]=$ $-0.1\left|\mathfrak{R}\left[\epsilon_{r 1}\right]\right|$. As shown in Figure 6(a), when $\epsilon_{r 1}<0$, that is, the film-slab is composed of a material with negative permittivity, the $\mathcal{E} \mathcal{F}$ attains large values at certain distinct locations of the superstrate's permittivity in the region $1<$ $\epsilon_{r 2}<5$. In particular, $\mathcal{E} \mathcal{F}$ exhibits some sharp resonances

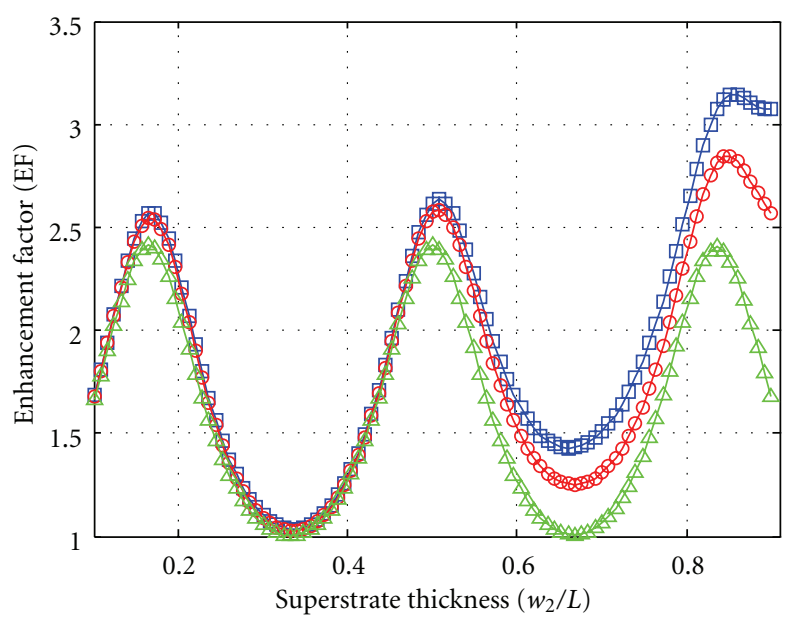

$$
\begin{array}{ll}
\square & \chi / \lambda_{0}=0.01 \\
\square & \chi / \lambda_{0}=0.05 \\
\triangle & \chi / \lambda_{0}=0.5
\end{array}
$$

(a)

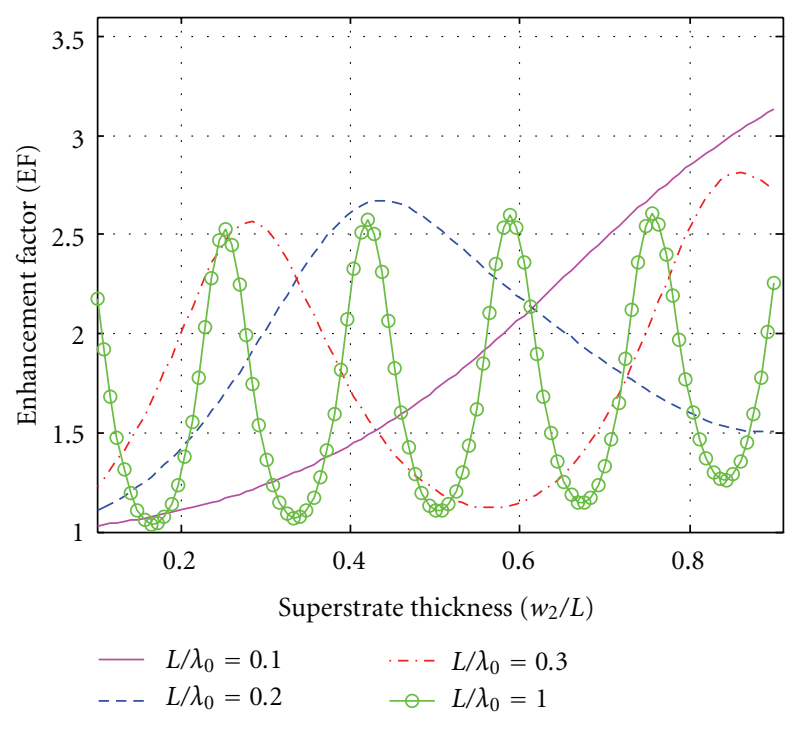

(b)

FigURE 4: Enhancement factor as function of the superstrate's relative thickness $w_{2} / L$ for $\epsilon_{r 1}=50-5 j, \epsilon_{r 2}=9, \mu_{r 2}=1$ with (a) $L=0.5 \lambda_{0}$ and $\chi / \lambda_{0}=0.01,0.05,0.5$ and (b) $\chi / \lambda_{0}=0.1$ and $L / \lambda_{0}=0.1,0.2,0.3,1$.

for a film-slab permittivity corresponding to an $\epsilon$-nearzero (ENZ) material $[19,20]$ or a low-index metamaterial (LIM) $[21,22]$. Such materials are known to possess also other remarkable EM properties, including controlling the radiation pattern, and tunneling of electromagnetic energy $[19,20]$. On the other hand, Figure 6(b) shows that sharp $\mathcal{E F}$ resonances with significantly large values occur for distinct negative values of the superstrate's permeability $\mu_{r 2}$ and for a film-slab with both positive as well as negative permittivity $\epsilon_{r 1}$. It is important to point out that $\mathcal{E} \mathcal{F}$ may take a value of the order of 50 for an ENZ film-slab, namely, 


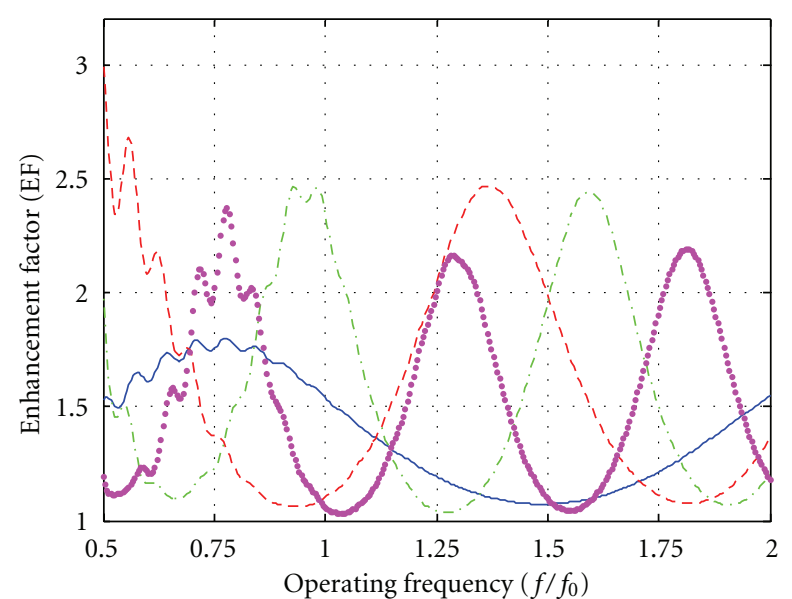

$\begin{array}{ll}\varepsilon_{r 2}=2 & -\cdot \varepsilon_{r 2}=10 \\ --\varepsilon_{r 2}=5 & -\varepsilon_{r 2}=15\end{array}$

(a)

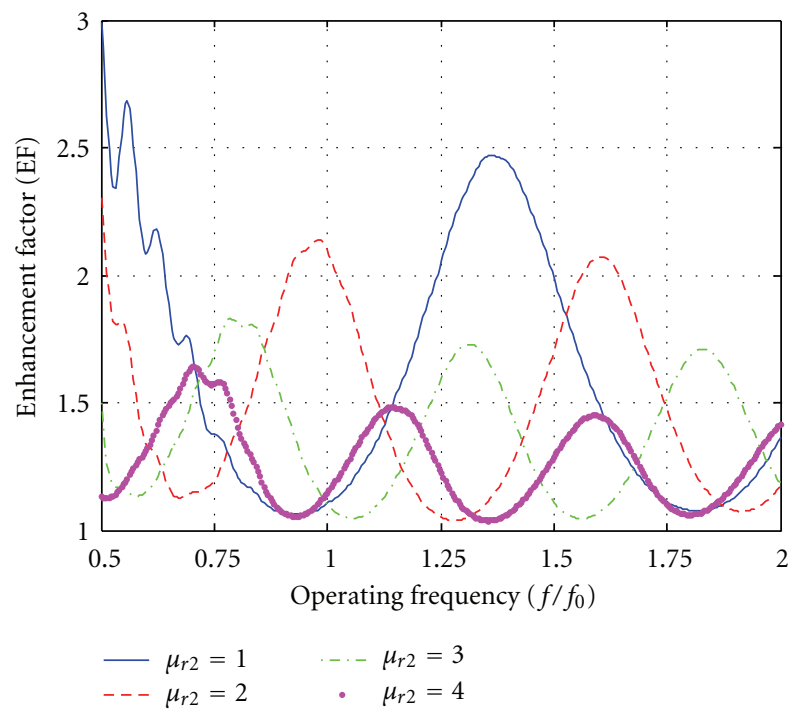

(b)

FIGURE 5: Enhancement factor as function of the structure's normalized operating frequency $f / f_{0}$ for $f_{0}=300 \mathrm{THz}, \epsilon_{r 1}=$ $50-5 j, w_{2}=0.25 \lambda_{0}$ with (a) $\mu_{r 2}=1$ and $\epsilon_{r 2}=2,5,10,15$ and (b) $\epsilon_{r 2}=5$ and $\mu_{r 2}=1,2,3,4$.

meaning that the induced total field inside an ENZ filmslab, covered by the specific superstrate, becomes 50 times the corresponding field in the same slab when the superstrate is absent.

Next, we depict in Figures 7(a) and 7(b), the visualization of the total electric field induced inside the film-slab when the superstrate is absent and present, respectively. It is evident that the electric field in the slab is considerably enhanced when it is covered by a suitable superstrate. This fact also verifies that the enhancement factor $\mathcal{E} \mathcal{F}$, defined according to (21), is an adequate measure in order to describe properly the induced field enhancement.

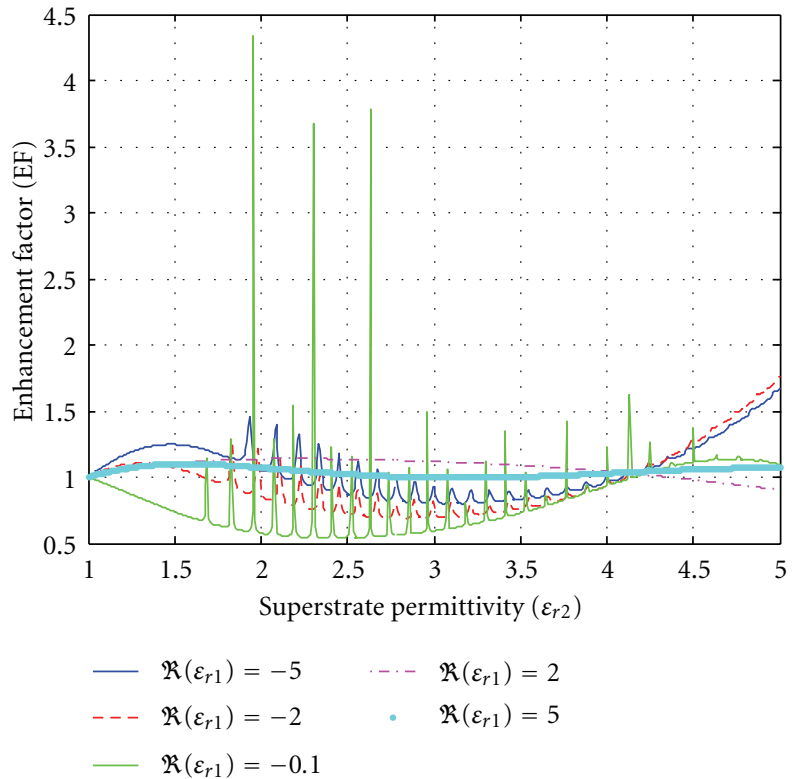

(a)

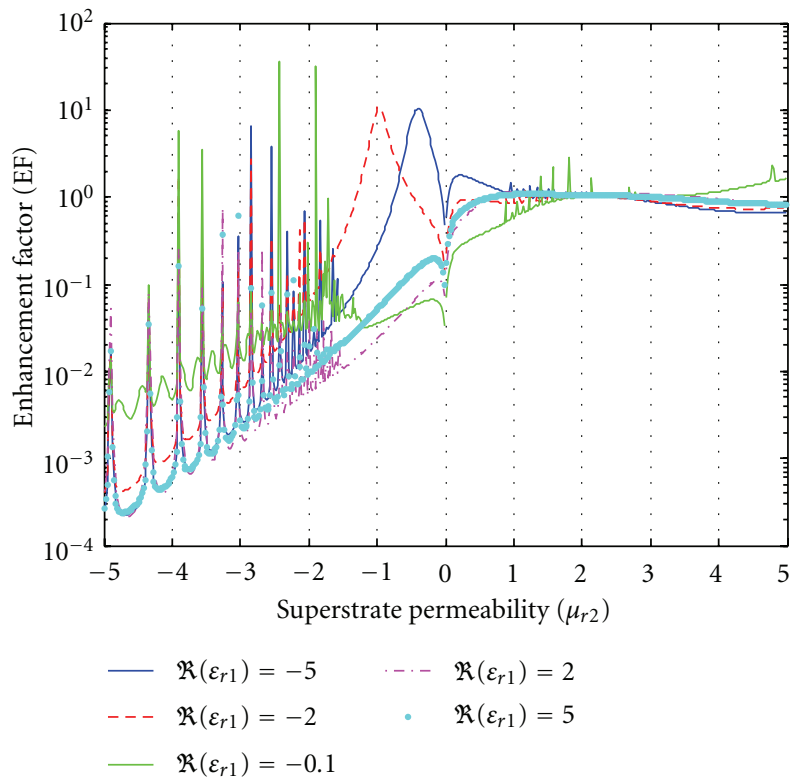

(b)

FIGURE 6: Enhancement factor as function of the superstrate's (a) relative permittivity $\epsilon_{r 2}$ for constant $\mu_{r 2}=1$ and (b) relative permeability $\mu_{r 2}$ for constant $\epsilon_{r 2}=2$, with $w_{2}=0.25 \lambda_{0}, \mathfrak{R}\left[\epsilon_{r 1}\right]=$ $-5,-2,-0.1,2,5$ and $\mathfrak{J}\left[\epsilon_{r 1}\right]=-0.1\left|\mathfrak{R}\left[\epsilon_{r 1}\right]\right|$.

Finally, we address the key issue concerning the physical mechanism of the achieved field enhancement. To this end, Figures $8(\mathrm{a})$ and $8(\mathrm{~b})$ show the visualization of the total electric field induced in the region $x \in \mathbb{R}, L-w_{2}<y<L$ when (a) no superstrate is present, and (b) the region is occupied by the specific superstrate, considered in Figure 7. Clearly, the field in the region under consideration is significantly reduced when the superstrate is present compared to when it is absent. This fact is directly related with the corresponding 


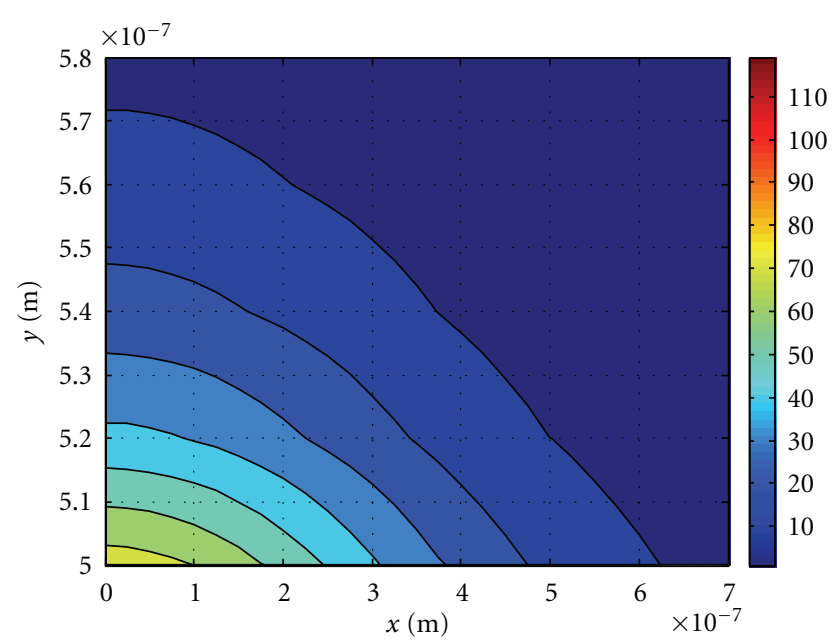

(a)

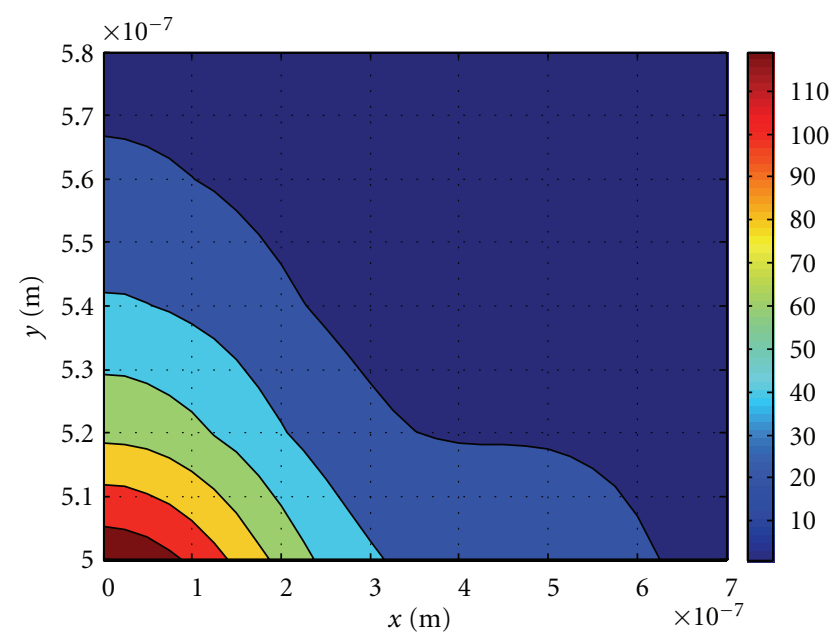

(b)

FIGURE 7: Contour plots of the total electric field induced inside the film-slab for $\epsilon_{r 1}=-5(1+0.1 i)$ and for (a) no superstrate and (b) superstrate with $\epsilon_{r 2}=1.94, \mu_{r 2}=1, w_{2}=0.25 \lambda_{0}$.

significant field enhancement observed inside the film-slab region when a suitable superstrate covers the slab, as depicted and discussed in Figure 7. Hence, the field is redistributed between the superstrate and film-slab regions in the sense that

(1) when no superstrate is present, then the total field

(i) in the region $x \in \mathbb{R}, L-w_{2}<y<L$ attains large values since this region is close to the beam's source and no intermediate layer exists causing reflections;

(ii) in the film-slab attains moderate values;

(2) when a suitable superstrate is present, then the total field

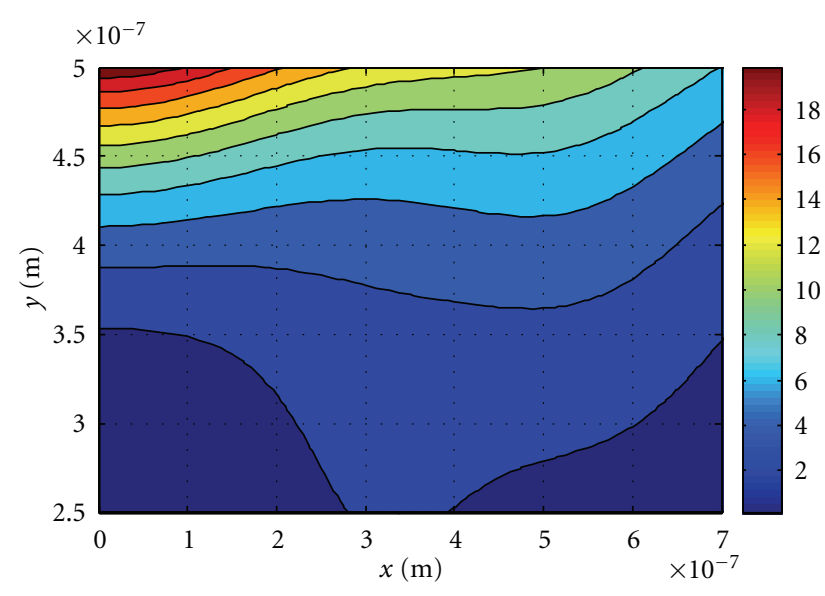

(a)

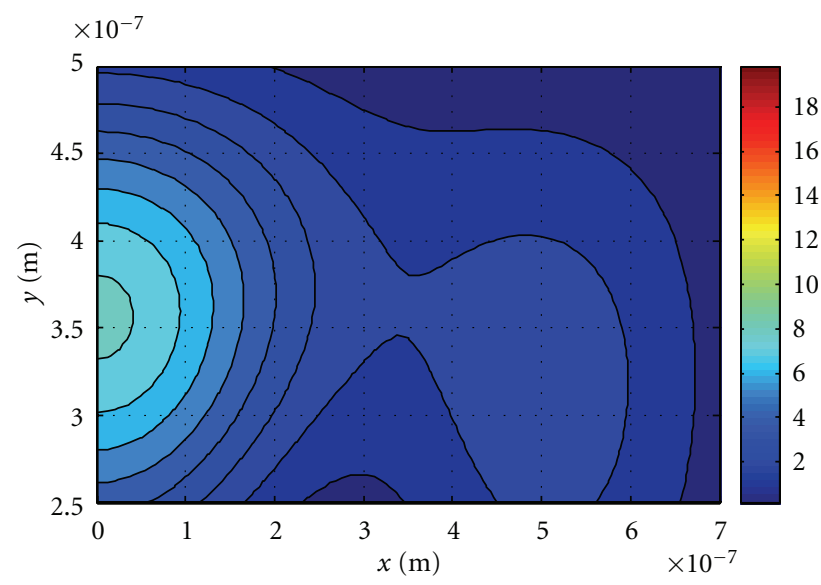

(b)

Figure 8: Contour plots of the total electric field induced in the region $x \in \mathbb{R}, L-w_{2}<y<L$ for the same set of constant parameters with Figure 7, when (a) no superstrate is present, and (b) the region is occupied by a superstrate with $\epsilon_{r 2}=1.94, \mu_{r 2}=1, w_{2}=0.25 \lambda_{0}$.

(i) in the film-slab is significantly enhanced precisely due to the presence of the specific superstrate;

(ii) in the region $x \in \mathbb{R}, L-w_{2}<y<L$ is considerably reduced compared to case 1 where the superstrate is absent.

\section{Conclusions}

We analyzed the Gaussian beam external excitation of a grounded dielectric slab configuration. The related boundary value problem was solved by analytical techniques. The main focus was given at the investigation of the device's potential application as an electromagnetic power concentrator by suitably altering the physical and geometrical parameters of an additional superstrate layer, lying on top of the device. We defined the "enhancement factor" representing the ratio of the total field's power induced in the film-slab when the superstrate is present over the corresponding power 
when the superstrate is absent. Numerical simulations were presented concerning the achieved enhancement factor of the slab configuration. It was demonstrated that it is possible to achieve large enhancement factor values by choosing properly the permittivity, the permeability, and the thickness of the superstrate layer. In particular, it was exhibited that the film-slab's field is significantly enhanced when the slab is composed by an $\epsilon$-near-zero or low-index metamaterial.

Interesting future work directions concern the conduction of a systematic investigation of the optimization of the superstrate's parameters in order to achieve the largest possible enhancement factor values for a given grounded slab configuration.

\section{References}

[1] Y. J. Zheng, H. Liu, S. M. Wang et al., "Selective optical trapping based on strong plasmonic coupling between gold nanorods and slab," Applied Physics Letters, vol. 98, no. 8, Article ID 083117, 2011.

[2] A. A. R. Neves, A. Fontes, L. Y. De Pozzo et al., "Electromagnetic forces for an arbitrary optical trapping of a spherical dielectric," Optics Express, vol. 14, no. 26, pp. 13101-13106, 2006.

[3] T. M. Grzegorczyk and J. A. Kong, "Analytical prediction of stable optical trapping in optical vortices created by three TE or TM plane waves," Optics Express, vol. 15, no. 13, pp. 80108020, 2007.

[4] E. Verhagen, L. Kuipers, and A. Polman, "Field enhancement in metallic subwavelength aperture arrays probed by erbium upconversion luminescence," Optics Express, vol. 17, no. 17, pp. 14586-14598, 2009.

[5] C. Vassallo, "Theory and practical calculation of antireflection coatings on semiconductor laser diode optical amplifiers," IEE Proceedings, vol. 137, no. 4, pp. 193-202, 1990.

[6] C. A. Valagiannopoulos, "High selectivity and controllability of a parallel-plate component with a filled rectangular ridge," Progress In Electromagnetics Research, vol. 119, pp. 497-511, 2011.

[7] J.-H. Choe, Q. H. Park, and H. Jeon, "Effect of metallic slab cladding on photonic crystal band structures," Journal of the Korean Physical Society, vol. 53, no. 5, pp. 2591-2595, 2008.

[8] A. Alù, D. Rainwater, and A. Kerkhoff, "Plasmonic cloaking of cylinders: finite length, oblique illumination and crosspolarization coupling," New Journal of Physics, vol. 12, Article ID 103028, 2010.

[9] C. A. Valagiannopoulos and N. L. Tsitsas, "Integral equation analysis of a low-profile receiving planar microstrip antenna with a cloaking superstrate," Radio Science, vol. 47, Article ID RS2022, 2012.

[10] C. A. Valagiannopoulos, "Electromagnetic scattering of the field of a metamaterial slab antenna by an arbitrarily positioned cluster of metallic cylinders," Progress in Electromagnetics Research, vol. 114, pp. 51-66, 2011.

[11] F. Yang, A. Aminian, and Y. Rahmat-Samii, "A novel surfacewave antenna design using a thin periodically loaded ground plane," Microwave and Optical Technology Letters, vol. 47, no. 3, pp. 240-245, 2005.

[12] G. D. Landry and T. A. Maldonado, "Gaussian beam transmission and reflection from a general anisotropic multilayer structure," Applied Optics, vol. 35, no. 30, pp. 5870-5879, 1996.
[13] E. E. Kriezis, P. K. Pandelakis, and A. G. Papagiannakis, "Diffraction of a Gaussian beam from a periodic planar screen," Journal of the Optical Society of America A, vol. 11, no. 2, pp. 630-636, 1994.

[14] J. Yang, L.-W. Li, K. Yasumoto, and C. H. Liang, "Twodimensional scattering of a gaussian beam by a periodic array of circular cylinders," IEEE Transactions on Geoscience and Remote Sensing, vol. 43, no. 2, pp. 280-285, 2005.

[15] P. H. Bolivar, M. Brucherseifer, J. G. Rivas et al., "Measurement of the dielectric constant and loss tangent of high dielectricconstant materials at terahertz frequencies," IEEE Transactions on Microwave Theory and Techniques, vol. 51, no. 4, pp. 1062 1066, 2003.

[16] A. Alù, A. Salandrino, and N. Engheta, "Negative effective permeability and left-handed materials at optical frequencies," Optics Express, vol. 14, no. 4, pp. 1557-1567, 2006.

[17] B. García-Cámara, F. Moreno, F. González, J. M. Saiz, and G. Videen, "Light scattering resonances in small particles with electric and magnetic propertie," Journal of the Optical Society of America A, vol. 25, no. 2, pp. 327-334, 2008.

[18] P.-Y. Chen, M. Farhat, and A. Alù, "Bistable and self-tunable negative-index metamaterial at optical frequencies," Physical Review Letters, vol. 106, no. 10, Article ID 105503, 2011.

[19] M. Silveirinha and N. Engheta, "Tunneling of electromagnetic energy through subwavelength channels and bends using $\mathcal{E}$ near-zero materials," Physical Review Letters, vol. 97, no. 15, Article ID 157403, 2006.

[20] A. Alù, M. G. Silveirinha, A. Salandrino, and N. Engheta, "Epsilon-near-zero metamaterials and electromagnetic sources: Tailoring the radiation phase pattern," Physical Review B, vol. 75, no. 15, Article ID 155410, 2007.

[21] G. Lovat, P. Burghignoli, F. Capolino, D. R. Jackson, and D. R. Wilton, "Analysis of directive radiation from a line source in a metamaterial slab with low permittivity," IEEE Transactions on Antennas and Propagation, vol. 54, no. 3, pp. 1017-1030, 2006.

[22] C. A. Valagiannopoulos, "Effect of cylindrical scatterer with arbitrary curvature on the features of a metamaterial slab antenna," Progress in Electromagnetics Research, vol. 71, pp. 5983, 2007. 

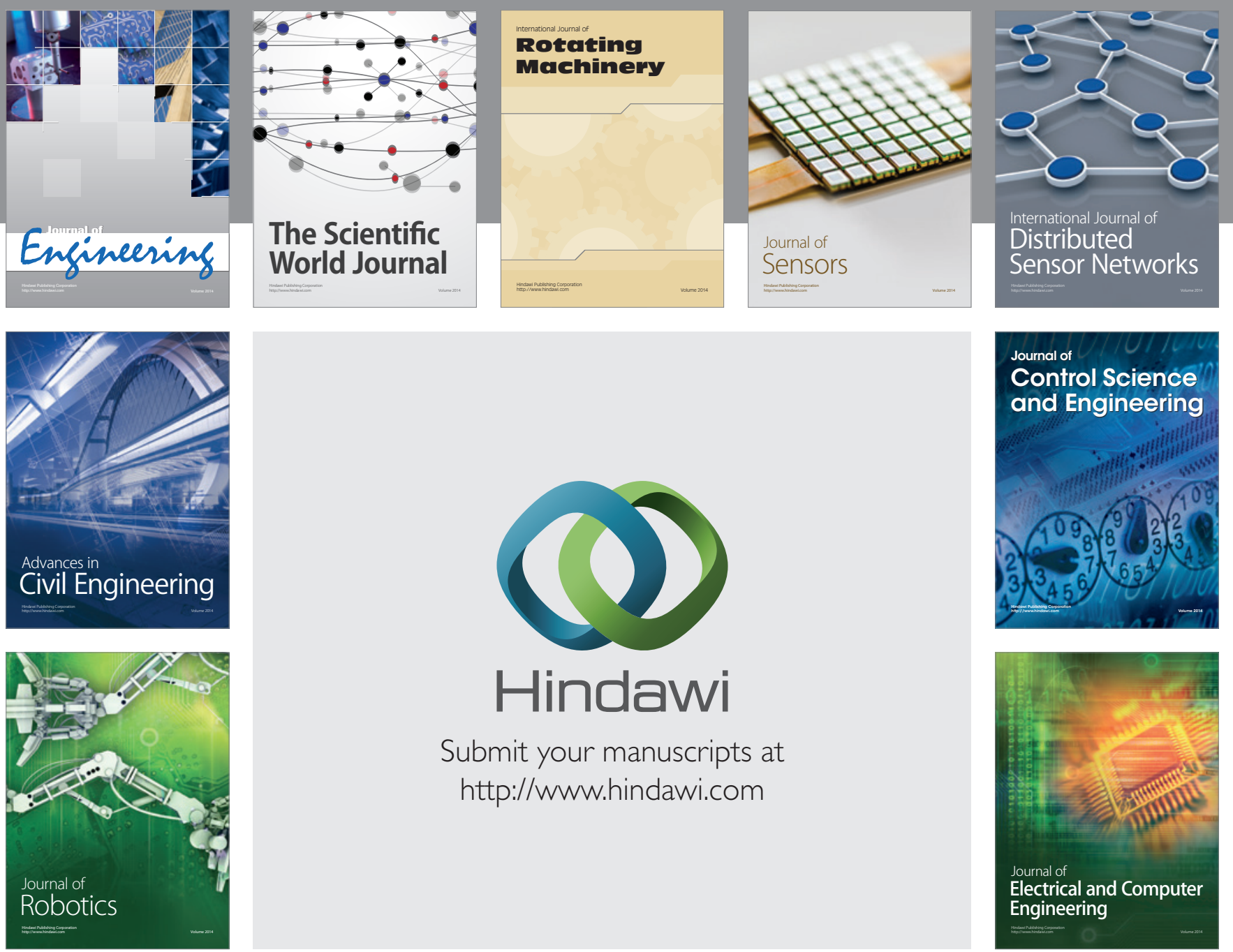

Submit your manuscripts at

http://www.hindawi.com
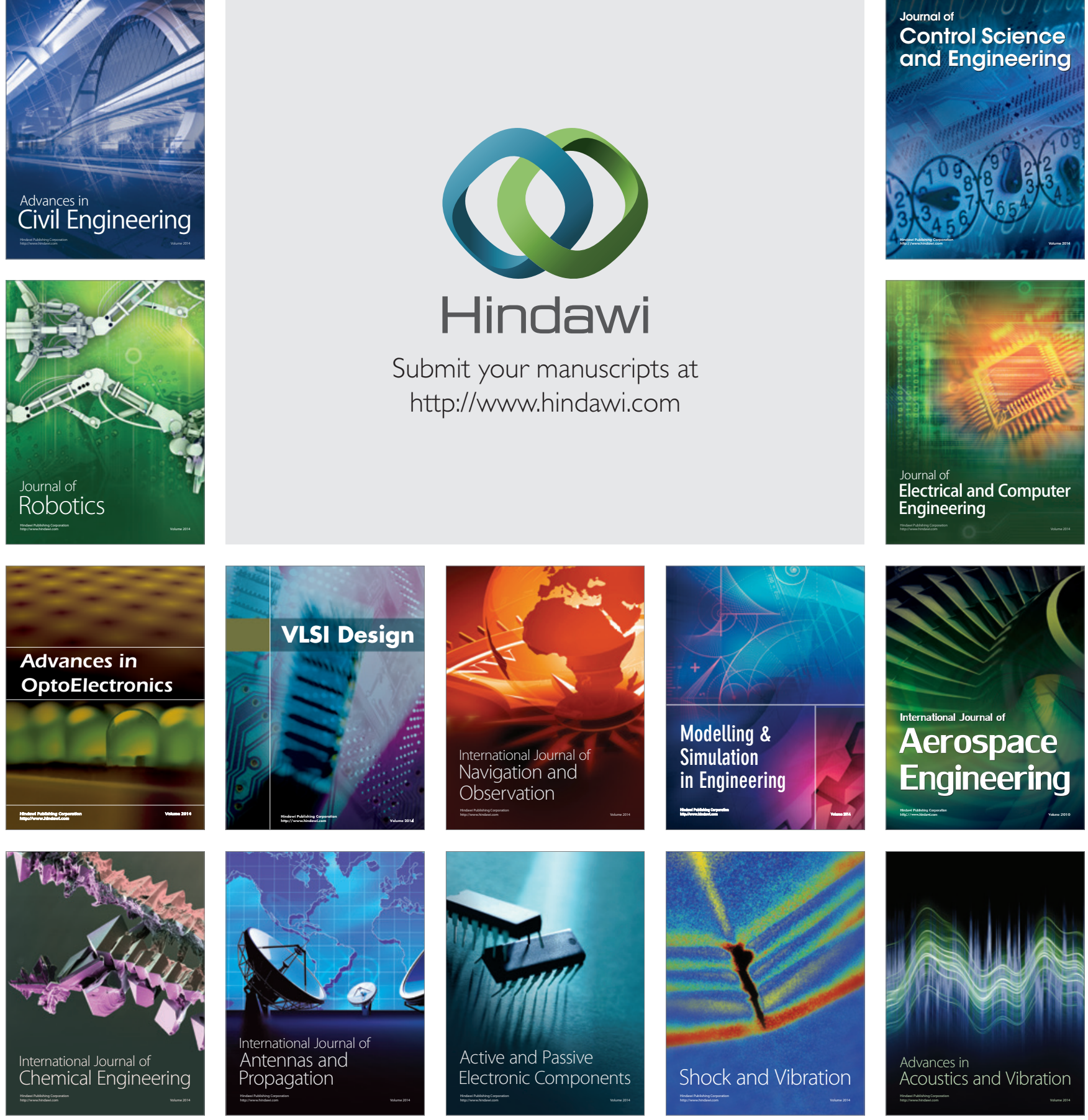